\title{
Fault-Tolerant Wormhole Routing Algorithms in Meshes in the Presence of Concave Faults
}

\author{
Seungjin Park \\ Dept. of Computer Science \\ Michigan Tech. University \\ Houghton, MI 49931, U.S.A. \\ spark@mtu.edu
}

\author{
Jong-Hoon Youn \\ Dept. of Computer Science \\ Oregon State University \\ Corvallis, OR 97330, U.S.A. \\ jhyun@cs.orst.edu
}

\author{
Bella Bose \\ Dept. of Computer Science \\ Oregon State University \\ Corvallis, OR 97330, U.S.A. \\ bose@cs.orst.edu
}

\begin{abstract}
A fault ring is a connection of only nonfaulty adjacent nodes and links such that the interior of the ring contains only faulty components. This paper proposes two wormhole routing algorithms that deal with more relaxed shapes of fault rings than previously known algorithms [1, 2, 3] in the mesh networks. As a result, the number of components to be made disabled would be reduced considerably in some cases. First algorithm, called F4, uses four virtual channels and allows all four sides of fault rings to contain concave shapes. Second algorithm, F3, permits up to three sides to contain concave shapes using only three virtual channels. Both F3 and F4 are free of deadlock and livelock and guarantee the delivery of messages between any pair of nonfaulty and connected nodes in the network.
\end{abstract}

\section{Introduction}

Since processors in a multicomputer network need to communicate with other, efficient communication is essential to enhance the performance of the system. The wormhole-routing switching has been dominant for its low-latency communication, and it has been adopted by numerous parallel machines.

Two important issues in designing routing algorithms are deadlock and livelock freedom. These properties are necessary for the guaranteed delivery of a message to its destination. Another issue in designing routing algorithm is the extent of the fault information available at each node. In routing strategy used in the paper, each node needs to know only the local fault information $[1,2,3,4]$. With this limited information the routing algorithms becomes simple, and thus the routing decision at each node becomes fast. However, the resulting path by the algorithms may not be the optimal.

A set of adjacent faults forms two types of fault shapes in mesh networks: fault-ring and fault-chain. If a connection of only nonfaulty adjacent nodes and links forms a ring such that the interior of the ring contains only faulty components, then the shape is called fault-ring $(f$ ring in short). When an f-ring touches one or more boundaries of the network, it becomes a fault-chain $(f$ chain in short).

An extensive amount of work has been done on faulttolerant routing in mesh networks [1, 2, 3, 4]. Among them, we briefly explain the most relevant works to our algorithms. Boppana and Chalasani [1] have proposed fault-tolerant routing algorithms in mesh networks using wormhole technique. Their algorithms, based on the ecube routing, are deadlock- and livelock-free using only local knowledge of faults. Both f-rings and f-chains are allowed in their fault model, however, the shape of f-rings is restricted to rectangle. Four virtual channels are used to implement the algorithm. Later Sui and Wang [3] have improved the algorithm using only three virtual channels. In [2] Boppana and Chalasani proposed an algorithm tolerating more general forms of f-rings, called nonconvex. Let $a, l_{1}$, and $l_{2}$ be any nonfaulty node and two faulty links of an f-ring. In nonconvex fault model, no nonfaulty node, $a$, exists between two faulty links, $l_{1}$ and $l_{2}$, in the same row (respectively, column) of the mesh.

While permitting arbitrary fault patterns may still be an elusive goal, it would be desirable to relax the restriction on the shape of the f-rings. To achieve this goal, we present a routing algorithm which tolerates more relaxed fault models than those in $[1,2,3]$ using four virtual channels. Further, we also show another algorithm using only three virtual channels for more restricted f-rings. Our algorithms are better than those in $[1,2,3]$ in terms of the number of processors that should be made faulty. Each nonfaulty node in the proposed algorithms may need to know the status of nodes beyond its neighbor nodes. In order to do this, it may need additional time. Even though we consider only nonoverlapped f-rings in this paper, the proposed algorithms can easily be extended to deal with $\mathrm{f}$ chains and overlaps by creating more virtual channels.

The rest of the paper is organized as follows. Section 2 describes the necessary information to understand the paper. Section 3 explains the new fault-tolerant algorithms. Conclusion is presented in Section 4. 


\section{Background}

In this section we depict the fault models that have been adopted in the design of the proposed algorithms. In our fault model, both node and link failures are considered, i.e., either the entire processor element (node) and its associated router or any communication line (link) could fail. It is also assumed that the faults do not disconnect the network.

A node in column $a$ and row $b$ is represented by $(a, b)$ in a 2D mesh. A link that connects node $(a, b)$ and node $(c$, $d)$ is represented by $(a, b) \leftrightarrow(c, d)$.

Based on the number of faulty links incident to a node, each node on an f-ring falls into one of the following three categories:

1) Convex node - no faulty link incident to it.

2) Concave node - exactly two faulty links incident to it.

3) Plain node - only one faulty link incident to it.

A portion of an f-ring that consists of two convex nodes with only plain nodes, if any, between them is called convex section. Likewise, if a portion consists of two concave nodes and possibly with some plain nodes in between them is called concave section. If a convex section of an f-ring is projecting towards the West (respectively, East, South, North) side of the f-ring, it is called West (respectively, East, South, North) convex section. To distinguish the two convex nodes in a convex section, we call them the upper, the lower, the left and the right convex nodes, respectively, according to their relative positions in the section.

In our fault models, there might be several f-rings. We divide each f-ring into four portions: the North, South, West and East sides. All nodes and links between the upper convex node of the upper-most West (respectively, East) convex section and the lower convex node of the lower-most West (respectively, East) convex section form the West (respectively, East) side. Note that there exists at least one East and one West convex sections. If there is only one West (respectively, East) convex section, the West (respectively, East) side is between upper and lower convex node of the section. The North (respectively, South) side is the northern (respectively, southern) portion between the East and West sides.

EXAMPLE 1: In Fig. 1, some of the convex nodes are (0, $2),(0,3),(1,4)$, some of the concave nodes are $(1,2),(1$, $3),(2,4),(2,5)$, and some of the plain nodes are $(3,7),(4$, $3),(6,2)$. $\{(1,5),(1,6),(1,5) \leftrightarrow(1,6)\}$ and $\{(2,7),(3,7)$ $(4,7),(2,7) \leftrightarrow(3,7),(3,7) \leftrightarrow(4,7)\}$ are some examples of convex section, and $\{(4,6),(5,6),(6,6),(4,6) \leftrightarrow(5$, $6),(5,6) \leftrightarrow(6,6)\}$ and $\{(2,4),(2,5),(2,4) \leftrightarrow(2,5)\}$ are some examples of concave section. $\{(8,3),(8,4),(8,5)$, $(8,3) \leftrightarrow(8,4),(8,4) \leftrightarrow(8,5)\}$ is an example of East convex section, and $\{(2,4),(2,5),(2,4) \leftrightarrow(2,5)\}$ is a West concave section. $(1,6)$ is the upper convex node of the upper-most convex section, and $(0,2)$ is the lower convex node of the lower-most convex section. Therefore, the portion from node $(0,2)$ to $(1,6)$ is the West side, from $(8,3)$ to $(8,5)$ is the East side, from $(1,6)$ to $(8,5)$ is the North side, and from $(0,2)$ to $(8,3)$ is the South side.

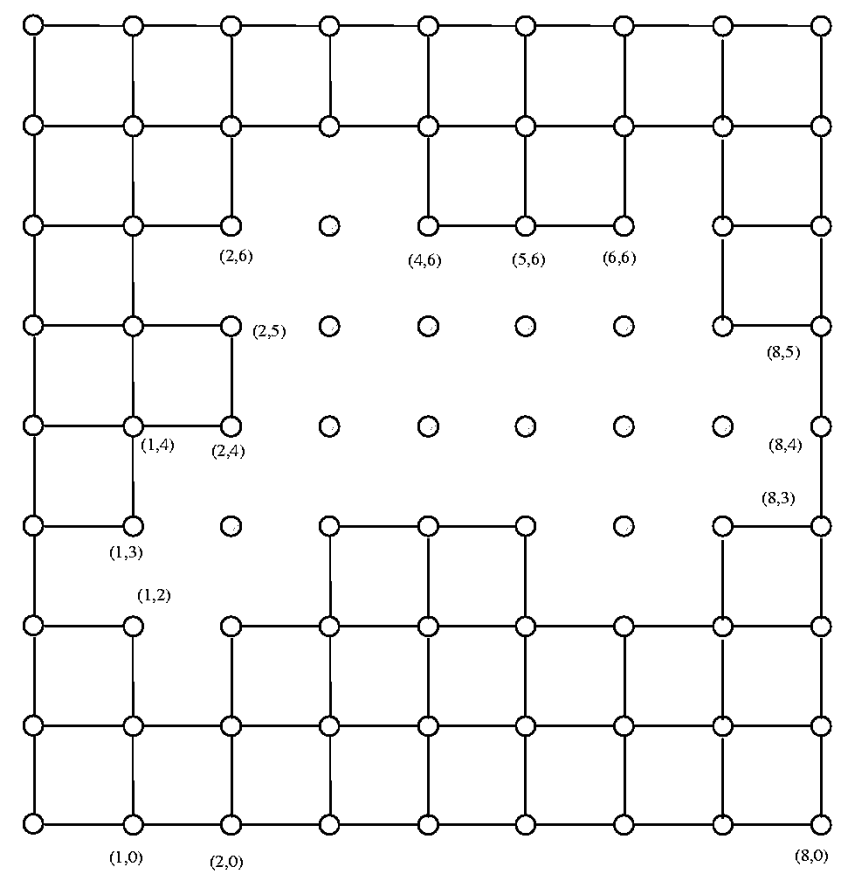

Figure 1. An example of an f-ring.

\subsection{Fault model}

Let us take a portion of a side of an f-ring and align it along the $x$-axis with the faults below it. Let $l_{1}=\left(x_{a}, y_{b}\right)$ $\leftrightarrow\left(x_{a}, y_{b+1}\right), l_{2}=\left(x_{c}, y_{d}\right) \leftrightarrow\left(x_{c}, y_{d+1}\right)$ be any two faulty links from the inside of the same f-ring. In our fault model, if $x_{a}=x_{c}$, every nodes between $l_{1}$ and $l_{2}$ are faulty.

For all nodes in the portion, if their $y$ values do not decrease (resp., increase) as $x$ values increase, the portion is called monotonically increasing (resp., monotonically decreasing). In Fig. 1, for example the portions from node $(1,6)$ to node $(4,7)$ and from node $(1,1)$ to node $(0$, $2)$ are monotonically increasing, and the portions from node $(7,2)$ to $(3,3)$ and from $(6,7)$ to $(8,5)$ are monotonically decreasing.

If a side is monotonically increasing first and then monotonically decreasing, then it is called convex side. Likewise, if a side is monotonically decreasing first and then monotonically increasing, then it is called concave side. If it consists of a combination of any number of monotonically increasing portion and monotonically decreasing portion, then it is called zigzag side. Note that a convex (or a concave) side is a zigzag side. 
As mentioned in Section 1, Chalasani and Boppana [1, 2] have proposed routing algorithms to handle the case in which each side of f-rings is only convex (it is called nonconvex in [2]). Examples of f-rings allowed in their algorithms are (a) and (b) in Fig. 2. However their algorithm may not work properly, if an f-ring contains some concave sides as shown in (c) and (d) of Fig 2. Therefore, if one wants to use the algorithms, concave sides should be made convex by converting some nonfaulty nodes to faulty. This results in the waste of costly resources. In this paper we propose a fault-tolerant routing algorithm which can tolerate f-rings with zigzag sides. Thus our algorithm works correctly for f-rings in Fig. 2 (a), (b), (c), and (d). However, Fig 2. (e) is not allowed in our algorithm because the North side of the fring is not a combination of only monotonically increasing and decreasing portions.

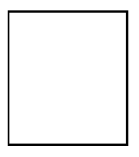

(a)

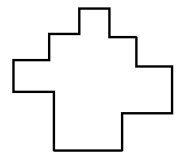

(b)

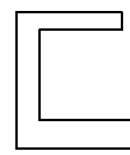

(c)

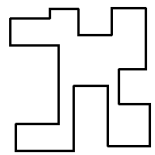

(d)

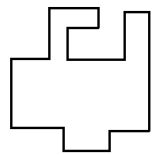

(e)
Figure 2. Several examples of f-rings.

\subsection{Formation of fault rings}

Messages are routed around the f-rings in two directions: clockwise and counter-clockwise. As shown in [1, 2, 3], only the fault status of the neighbor nodes is enough for any node to determine its two neighbor nodes on the f-ring in each direction. For example, in Fig. 1, since south link of node $(2,4)$ and east link of $(1,3)$ are faulty, it can be easily determined that the neighbor nodes of $(1,4)$ in counter-clockwise and clockwise directions are $(1,3)$ and $(2,4)$, respectively. These are also called left and right nodes, respectively. Refer to [2] for full description of the f-ring formation algorithm.

Each node in f-rings can easily be determined whether it is convex, concave or plain by the number of faulty links incident to it.

\subsection{Node position on an f-ring}

In addition to the knowledge about the neighbor nodes on f-rings, each node in our f-ring models has to know its position on the f-ring, i.e., which side it resides: North, South, East or West. Since an f-ring is a ring, every node on an f-ring has exactly two incident links on the f-ring. Let $X_{a b}$ indicate a convex node with links in $a$ and $b$ directions, where $a, b \in\{\mathrm{W}($ est), $\mathrm{E}$ (ast), S(outh), $\mathrm{N}$ (orth) $\}$. Likewise, $V_{a b}$ indicates a concave node. In the rest of the section, a node indicates a convex or concave node only, unless otherwise specified. Below we briefly describe the distributed algorithm for each node to attain its position information on the f-ring.

Step 1: Every node in the f-ring sends its incident f-ring link information to its neighbor nodes in both directions.

Step 2: Each node receives information from both neighbor nodes and determines, if possible, its position on the f-ring using the rules given in Table 1. For example, suppose the type of current node is $X_{E S}$ and its left node is $X_{E N}$, then the two nodes and all plain nodes, if any, between them form a West convex section. Thus, in our fault model all convex, concave and plain nodes in this section are in the West side. Note that only the nodes between the same type, i.e., between convex and convex or between concave and concave, know their positions after Step 2.

Step 3. Nodes between different types, namely convex and concave, determine their positions. Suppose a node, $v$ of type say convex, knows its position, $p \in\{\mathrm{W}, \mathrm{E}, \mathrm{N}$, $\mathrm{S}\}$, this may be due to its left neighbor node, $u$, is of the same type-in this case convex. Furthermore suppose $w$ is the nearest node on the right side of $v$, but not necessarily a neighbor, that knows its position $q \in\{\mathrm{W}, \mathrm{E}, \mathrm{N}, \mathrm{S}\}$. Let $V$ be all nodes between $v$ and $w$, including the plain nodes. Now node $v$ and $w$ set the position of nodes in $V$ as follows:

1) If $p=q$, then all nodes in $V$ are assigned the same position $p$.

2) If $p \neq q$, then one of $p$ or $q$ must be $\mathrm{N}$ or $\mathrm{S}$. Let us assume $\mathrm{p} \in\{\mathrm{N}, \mathrm{S}\}$. Then, all nodes in $V$ are assigned $p$. It can be easily verified that if $p \neq q$, then $p$ and $q$ cannot have the values only from $\{\mathrm{N}, \mathrm{S}\}$ or only from $\{\mathrm{E}, \mathrm{W}\}$.

\begin{tabular}{|c|c|c|}
\hline Node type & Neighbor node type & Position \\
\hline \hline$X_{E S}$ & Left node is $X_{E N}$ & West \\
\hline$X_{E S}$ & Right node is $X_{W S}$ & North \\
\hline$X_{W S}$ & Left node is $X_{E S}$ & North \\
\hline$X_{W S}$ & Right node is $X_{W N}$ & East \\
\hline$X_{W N}$ & Left node is $X_{W S}$ & East \\
\hline$X_{W N}$ & Right node is $X_{E N}$ & South \\
\hline$X_{E N}$ & Left node is $X_{W N}$ & South \\
\hline$X_{E N}$ & Right node is $X_{E S}$ & West \\
\hline$V_{E S}$ & Left node is $V_{E N}$ & East \\
\hline$V_{E S}$ & Right node is $V_{W S}$ & South \\
\hline$V_{W S}$ & Left node is $V_{E S}$ & South \\
\hline$V_{W S}$ & Right node is $V_{W N}$ & West \\
\hline$V_{W N}$ & Left node is $V_{W S}$ & West \\
\hline$V_{W N}$ & Right node is $V_{E N}$ & North \\
\hline$V_{E N}$ & Left node is $V_{W N}$ & North \\
\hline$V_{E N}$ & Right node is $V_{E S}$ & East \\
\hline
\end{tabular}

Table 1. Position of each node on an f-ring. 


\section{Routing algorithms}

In this paper we follow the convention and notation used in $[1,2]$. Let $M$ denote a message to be routed from the current node, $\left(a_{c}, b_{c}\right)$, to the destination node $\left(a_{d}, b_{d}\right)$. Our algorithms are based on the e-cube routing, in which all messages are routed in two phases: in the first phase the message is routed along $d_{0}$ dimension (row dimension) until $\left(a_{c}=a_{d}\right)$, and in the second phase it is routed along $\mathrm{d}_{1}$ dimension (column dimension) to the destination. $\mathrm{M}$ is said to be row message if it is in its first phase and column message, otherwise. Further, row messages traveling from West to East (respectively, East to West) are WE (respectively, $E W$ ) messages. Likewise, column messages traveling from North to South (respectively, South to North) are $N S$ (respectively, $S N$ ) messages. A row message can be changed to column message, but not vice versa in the e-cube routing.

Our first fault-tolerant routing algorithm, $F 4$, requires four virtual channels and handles the fault rings with zigzag sides. We also propose another algorithm, F3, which, using only three virtual channels, routes a message in a mesh with f-rings containing at most three zigzag sides. There is no limit on the number of faults in both algorithms. Note that neither overlapped f-rings nor fchains are allowed in our algorithms.

\subsection{F4: A routing algorithm for fault rings with four zigzag sides}

Let $\left(a_{c}, b_{c}\right)$ be the current host node of the given message M. If $\left(a_{c}, b_{c}\right)=\left(a_{d}, b_{d}\right)$, then $\mathrm{M}$ has reached its destination and so it is consumed. Otherwise, M starts as either WE or EW message depending on its value of $d_{0}$ dimension in source and destination addresses. Once $\left(a_{c}=a_{d}\right)$, M changes to column message and continues to travel towards its destination. $M$ travels in the network based on the e-cube algorithm until it reaches an f-ring. Once it reaches the f-ring, two things can happen:

1. If its e-cube hop is on the f-ring, then it continues to travel in the f-ring, or

2. If its e-cube hop is blocked, then it is misrouted.

In both cases, the message traveling on an f-rings can use only the following virtual channels depending on the message type: WE message uses $c_{0}$, EW message uses $c_{1}$, NS message uses $c_{2}$, and $S N$ message uses $c_{3}$ channel. The next hop for $\mathrm{M}$ is determined by the set of rules given in Table 2. The Procedure Set-Status sets the message status to normal or misrouted depending on the availability of the next e-cube hop. That is, if the next ecube hop for $\mathrm{M}$ is not blocked by a fault, then its status is set to normal, and $\mathrm{M}$ is routed using the e-cube algorithm. Otherwise it is misrouted.

If a message takes an e-cube hop on a link that is not on an f-ring, it can use any virtual channel without causing deadlock. This is because the e-cube routing needs only one virtual channel per physical channel [2].

Whenever M's status becomes misrouted, its direction on the f-ring is determined by the Procedure SetDirection using Table 3. Once its direction on the current f-ring is set, it will never be changed until $M$ leaves the $\mathrm{f}$ ring. Table 3 is established by the following rules in order to avoid deadlock.

1. When a WE message reaches the West side of an fring, route the message in counter clockwise direction if the destination is to the South of current node; otherwise route in clockwise direction. Note that the WE message never reaches the East side of the f-ring.

2. When a WE message reaches the North (resp., South) side of an f-ring, route the message clockwise (resp., counter clockwise) along the f-ring.

3. When a NS message reaches the North side of an fring, route the message in either direction.

4. When a NS message reaches the West side (resp., East) of an f-ring, route the message in counter clockwise (resp., clockwise) direction on the f-ring.

Similar rules are applied for EW and SN messages and are summarized in Table 3.

Procedure Set-Direction (M)

IF direction of $M$ is not null, return.

Otherwise, set direction according to Table 3.

Procedure Set-Status (M)

IF ( $\mathrm{M}$ is row message, and the next available e-cue hop is not on the f-ring), THEN set the status of $M$ to normal and the direction of $\mathrm{M}$ to null.

IF ( $\mathrm{M}$ is NS message, $a_{c}=a_{d}, b_{c}>b_{d}$, and the next e-cube hop is available), THEN set the status of $M$ to normal and the direction of $\mathrm{M}$ to null.

IF ( $\mathrm{M}$ is SN message, $a_{c}=a_{d}, b_{c}<b_{d}$, and the next ecube hop is available), THEN set the status of $M$ to normal and the direction of $\mathrm{M}$ to null.

Otherwise, set the status of $M$ to misrouted and setDirection (M).

Procedure Route-Message (M)

1 IF $\left(a_{c}=a_{d}\right)$ and $\left(b_{c}=b_{d}\right)$, THEN $M$ reaches its destination. Consume $\mathrm{M}$ and return.

2 IF $M$ is a row message and $\left(a_{c}=a_{d}\right)$, THEN change its type to SN if $\left(b_{d}>b_{c}\right)$ or NS if $\left(b_{c}>b_{d}\right)$.

3 SET-STATUS (M).

4 IF $M$ is normal, use the e-cube hop.

Otherwise, route $\mathrm{M}$ on the fault ring in the specified direction.

Table 2. Fault-tolerant algorithm, F4. 


\begin{tabular}{|c|c|c|}
\hline $\begin{array}{c}\text { Node } \\
\text { position }\end{array}$ & $\begin{array}{c}\text { Message } \\
\text { Type }\end{array}$ & $\begin{array}{c}\text { Misrouted } \\
\text { Direction }\end{array}$ \\
\hline \multirow{4}{*}{ North } & WE & Clockwise $(\mathrm{CW})$ \\
\cline { 2 - 3 } & $\mathrm{EW}$ & Counter Clockwise $(\mathrm{CCW})$ \\
\cline { 2 - 3 } & $\mathrm{NS}$ & Either CW or CCW \\
\cline { 2 - 3 } South & $\mathrm{SN}$ & $\mathrm{CW}$ if $a_{c}<a_{d}, \mathrm{CCW}$ if $a_{c}>a_{d}$ \\
\cline { 2 - 3 } & $\mathrm{WE}$ & $\mathrm{CCW}$ \\
\cline { 2 - 3 } & $\mathrm{EW}$ & $\mathrm{CW}$ \\
\cline { 2 - 3 } & $\mathrm{NS}$ & $\mathrm{CW}$ if $a_{c}>a_{d}, \mathrm{CCW}$ if $a_{c}<a_{d}$ \\
\hline \multirow{4}{*}{ West } & $\mathrm{WE}$ & $\begin{array}{c}\text { CCW if } b_{c}>b_{d}, \mathrm{CW} \text { if } b_{c}<b_{d}, \\
\text { otherwise either CW or CCW }\end{array}$ \\
\cline { 2 - 3 } & $\mathrm{EW}$ & $\mathrm{N} / \mathrm{A}$ \\
\cline { 2 - 3 } & $\mathrm{NS}$ & $\mathrm{CCW}$ \\
\cline { 2 - 3 } & $\mathrm{SN}$ & $\mathrm{CW}$ \\
\hline \multirow{4}{*}{ East } & $\mathrm{WE}$ & $\mathrm{N} / \mathrm{A}$ \\
\cline { 2 - 3 } & $\mathrm{EW}$ & $\begin{array}{c}\text { CW if } b_{c}>b_{d}, \mathrm{CCW} \text { if } b_{c}<b_{d}, \\
\text { otherwise either CW or CCW }\end{array}$ \\
\cline { 2 - 3 } & $\mathrm{NS}$ & $\mathrm{CW}$ \\
\cline { 2 - 3 } & $\mathrm{SN}$ & $\mathrm{CCW}$ \\
\hline
\end{tabular}

Table 3. Direction to be set for the misrouted messages on f-rings.

EXAMPLE 2: Consider the F4 routing of a message $M$ from $(1,7)$ to $(5,0)$ in Fig. 3. M uses $c_{0}$ channel from $(2$, $7)$ to $(4,5)$, and uses $c_{2}$ from $(5,3)$ to $(5,1)$. At first, $M$ is a normal WE message and is routed to $(3,7)$. Since its e-cube hop is blocked by the faulty link $(3,7) \leftrightarrow(4,7)$, its status is set to misrouted at $(3,7)$. Because M's destination is south of $(3,7)$, its direction is set to the counter clockwise orientation and is routed along (3, $6) \rightarrow(2,6) \rightarrow(2,5)$. At $(2,5) \mathrm{M}$ becomes normal message again and travels up to $(5,5)$. $M$ becomes a column (NS) message at $(5,5)$, and travels to the south along (5, $5) \rightarrow(5,4) \rightarrow(5,3)$ as a normal message. At $(5,3)$ M's ecube hop is blocked by the faulty link $(5,3) \leftrightarrow(5,2)$. Thus, its status is set to misrouted at $(5,3)$ and misrouted in the counter clockwise orientation (the orientation is chosen randomly) along the f-ring to $(5,1)$. At $(5,1) \mathrm{M}$ is set to a normal NS message, since the destination and current node are located on the same column and its ecube hop is not blocked. $\quad \mathrm{M}$ is routed to $(5,1) \rightarrow(5,0)$ as a normal NS message. Note that the hops from $(5,1)$ to $(5$, $0),(4,5)$ to $(5,5),(5,5)$ to $(5,4)$, and $(5,4)$ to $(5,3)$ are not on the f-ring. Therefore, as mentioned above, any of the four classes of virtual channels may be used for the hop.

We now prove the deadlock freedom in $\mathrm{F} 4$.

Lemma 1: Let $m_{1}, m_{2}$ and $m_{3}$ be the messages using the same virtual channels. Also let $n_{1}, n_{2}$ and $n_{3}$ be the nodes in f-rings $f_{1}, f_{2}$ and $f_{3}$, respectively. If $m_{1}$ travels from $n_{1}$ to $n_{2}$, and $m_{2}$ travels from $n_{2}$ to $n_{3}$, then there is a message $m_{3}$ that travels from $n_{1}$ to $n_{3}$.

Lemma 1 implies that any deadlock involving multiple f-rings can be checked by just considering between two frings.

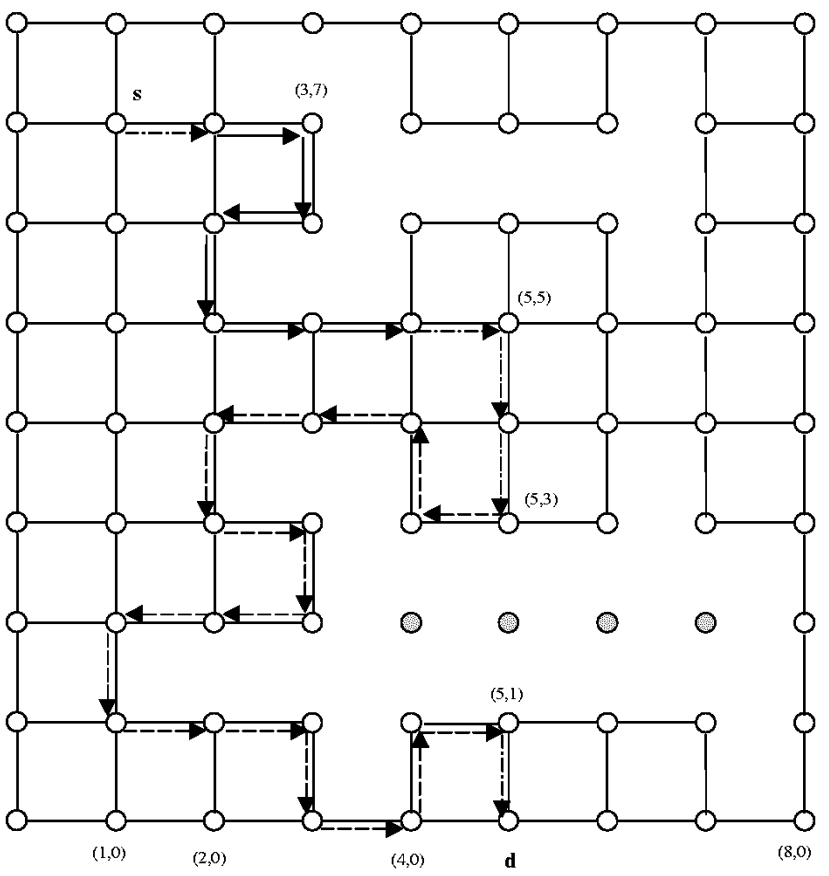

Figure 3. An example of F4 using four virtual channels. Solid arrows indicate the virtual channel $c_{0}$, and the dotted arrows indicate the virtual channel $c_{2}$. The other type of arrow is used for the e-cube hops that are not on the fring, and it can be any virtual channel.

Theorem 1: F4 algorithm causes no deadlock in 2D meshes that contain any number of $f$-rings with zigzag sides.

Proof: In F4 each message type uses an exclusive set of virtual channels for its travel to the destination. Row messages may change their types into column messages, but not vice versa. Further, WE messages cannot be changed into EW messages, and vice versa. Likewise, NS messages cannot be changed into SN messages, and vice versa. Thus, deadlock involving more than one type of messages cannot occur. Hence, to prove the deadlock freedom, it is sufficient to show that there is no deadlock among messages of a specific type.

In the following we provide the proof of deadlock freedom for WE messages ( $c_{0}$ channels) only. Deadlock freedom for other message types can be proved in a similar manner. Our proof consists of two parts: no cyclic dependency among WE messages 1) in an f-ring, and 2) involving multiple f-rings. 
1) Deadlock freedom with WE messages in an f-ring: According to F4, WE messages can travel either clockwise or counterclockwise on f-rings, and it can be easily shown that these two types of messages do not share the same link. Thus, no deadlock can occur in an fring.

2) Deadlock freedom with WE messages involving multiple f-rings: Suppose a WE message travels along the part, say $p_{1}$, of $f_{1}$ and reaches at the node $b$ on $f_{2}$. In order to form a cycle, there must be a WE message that travels through $b$ and reaches $p_{l}$. We prove the theorem by showing the non-existence of such a message. In the following we consider all possible cases that can occur with WE messages.

Case 1) Node $\mathrm{b}$ is at the North or South side of $f_{2}$.

According to F4, the message will never take EW channels. Therefore, it never reaches $p_{l}$. (It may reach other parts of $f_{1}$ again if any part of $f_{2}$ locates in the south concave section or the north concave section of $f_{l}$, but not $\left.p_{1}\right)$.

Case 2) Node $b$ is at the West side of $f_{2}$. If the West side contains no concave section, a similar argument as in Case 1 above can be applied. If $f_{2}$ does contain concave sections, the WE message may travel clockwise (resp., counter clockwise) taking some EW channels along $f_{2}$ until it reaches west-most node, say $d$, of North side (resp., South side). Note that since overlapping of fault rings are not allowed, the path from $b$ to $d$ cannot reach $p_{l}$. After reaching node $d$, Case 1 can be applied.

Also, F4 does not allow WE messages to reverse its direction on a column.

Similar argument can be applied to EW, NS and SN messages. Thus, there is no deadlock in F4.

\subsection{F3: A routing algorithm for f-rings with up to three zigzag sides using three virtual channels}

To implement the virtual channels, extra hardware such as flit buffers and proper logic is needed. Besides, these virtual channels are usually implemented on physical channel by using time sharing strategy. Therefore, a less number of virtual channels may lead to a better performance of the system.

In this section we propose another fault tolerant routing algorithm for f-rings with at most three zigzag sides. To satisfy this property, we make either all West sides or all East sides of f-rings be of convex fault model. In the previous routing algorithm, F4, it can be observed that only half of the $c_{0}$ (respectively, $c_{l}$ ) virtual channel links in f-rings is used by WE (respectively, EW) messages. For full utilization of the channel capacity, the WE and the EW messages share the same virtual channel, $c_{0}$, in our modified algorithm, F3. Consequently, the channel usage by $\mathrm{F} 3$ is: 1) the $\mathrm{WE}$ and $\mathrm{EW}$ messages use $c_{0}$ channel, 2) the NS messages use $c_{l}$, and 3) the $\mathrm{SN}$ messages use $c_{2}$ channel. Except these channel assignment to message types, F3 is same as F4.

Theorem 2: $F 3$ causes no deadlock in 2D meshes.

Proof: The same argument can be applied as shown in Theorem 1 for deadlock in an f-ring. Suppose two f-rings, $f_{1}$ and $f_{2}$ are involved in deadlock. Without loss of generality, assume that the location of $f_{1}$ is left of $f_{2}$. It can be seen that the only possible palces for deadlock between the two f-rings are East side of $f_{2}$ and West side of $f_{2}$. Let us assume that East sides of f-rings are convex. To satisfy the deadlock situation, the messages involved in the deadlock should have four or six "turns" in their paths [5]. However, since East side is concave, it is not possible to have "up-right" and "down-right" turns. Therefore, no deadlock occurs in F3.

In both F3 and F4, messages are misrouted only when they are blocked by f-rings. Otherwise, they proceed towards their desinations using the e-cube algorithm. Since there is a limited number of f-rings in the network, the amount of misrouted steps are also limited. Therefore, every message will eventually reach its destination without any livelock.

\section{Conclusion}

In this paper we have proposed two fault-tolerant wormhole routing algorithms in mesh networks. Both algorithms relax the restrictions in [1, 2, 3] to some extend. As a result, the number of components to be made disabled would be reduced considerably in some cases. First algorithm, F4, using four virtual channels, can tolerate any number of convex and concave portions in each side. Second algorithm, F3, uses only three virtual channels to tolerate fault rings with up to three zigzag sides. Both F3 and F4 are free of deadlock and livelock and guarantee the delivery of messages between any pair of nonfaulty and connected nodes in the network.

\section{References}

[1] R.V. Boppana and S. Chalasani, "Fault-Tolerant Wormhole Routing Algorithms for Mesh Networks," IEEE Trans. Computers, vol. 44, no. 7, pp. 848-864, July 1995.

[2] S. Chalasani and R.V. Boppana, "Communication in Multicomputers with Nonconvex Faults," IEEE Trans. Computers, vol. 46, no. 5, pp. 616-622, May 1997.

[3] P. Sui and S. Wang, "An Improved Algorithm for FaultTolerant Wormhole Routing in Meshes," IEEE Trans. Computers, vol. 46, no. 9, pp. 1040-1042, Sept. 1997.

[4] A. Chien and J. Kim, "Planar-Adaptive Routing: Low-Cost Adaptive Networks for Multiprocessors," Proc., $19^{\text {th }}$ Ann., Int'lSymp. Computer Architecture, pp. 268-277, 1992.

[5] C. J. Glass and L. M. Ni, "The turn model for adaptive routing", Proceedings of the $19^{\text {th }}$ International Symposium on Computer Architecture, pp. 278-287, May 1992. 